\title{
Avaliação da qualidade da consulta de enfermagem à criança no Programa de Saúde da Família*
}

\author{
Assessment of the quality of pediatric nursing consultation in the Family Health Program \\ Evaluación de la calidad de la consulta de enfermería al niño en el Programa de Salud de la \\ Familia
}

\section{Eliana Campos Leite Saparolli ${ }^{1}$, Nilce Piva Adami²}

\section{RESUMO}

Objetivo: Este estudo descritivo objetivou avaliar o processo desenvolvido nas consultas de enfermagem prestadas às crianças até um ano de idade em Unidades Básicas de Saúde no Programa de Saúde da Família do Município de São Paulo, considerando os conhecimentos técnico-científicos aplicados na implementação das ações básicas de saúde propostas pelo Ministério da Saúde. O referencial teórico foi ancorado na abordagem de processo da concepção sistêmica da avaliação de serviços de saúde. Métodos: Foi realizada a observação sistematizada de 114 consultas de enfermagem prestadas às crianças por 14 enfermeiras, utilizando-se um instrumento, contemplando critérios avaliativos validados por 11 especialistas. Resultados: Nas consultas observadas, as enfermeiras implementaram essas ações de forma adequada, carecendo, no entanto, de complementações. Conclusão: O processo assistencial, embora desenvolvido adequadamente, requer ajustes de fácil resolução em alguns procedimentos, a fim de abranger aspectos essenciais à saúde da criança nas unidades selecionadas do Programa de Saúde da Família.

Descritores: Avaliação de serviços de saúde; Serviços básicos de saúde; Qualidade da assistência à Saúde; Saúde da criança (Saúde Pública); Saúde da família; Referência e consulta

\begin{abstract}
Objective: This descriptive study has objectified to evaluate the process developed in the nursing consultations given to the children up to one year of age in Basic Units of Health of the Family Health Program of São Paulo City, considering the application of technical scientific knowledge in the implementation of the basic actions of health proposed for the Ministry of Health. The theoretical reference adopted was based on systemic conception of the evaluation of health services. Method: It was carried through systemize comment of 114 nursing consultations given to the children, for 14 nurses, using itself an instrument, contemplating evaluated criterion been valid for 11 specialists. Results: In the observed consultations, nurses had implemented these actions of adjusted form, lacking, however, of complementation. Conclusions: The care process, even so developed adequately, requires adjustments of easy resolution in some procedures, so as to enclose essential aspects to the children health in the selected Units of the Family Health Program.

Keywords: Health services evaluation; Basic health services; Quality of health care; Child health (Public Health); Family health; Referral and consultation

\section{RESUMEN}

Objetivo: Este estudio descriptivo tuvo como objetivo evaluar el proceso desarrollado en las consultas de enfermería prestada a niños de hasta un año de edad en Unidades Básicas de Salud del Programa de Salud de la Familia del Municipio de Sao Paulo, considerando los conocimientos técnico-científicos aplicados en la implementación de las acciones básicas de salud propuestas por el Ministerio de Salud. El referencial teórico estuvo anclado en el abordaje de proceso de la concepción sistémica de la evaluación de servicios de salud. Métodos: Se realizó la observación sistematizada de 114 consultas de enfermería a niños, desarrollada por 14 enfermeras, utilizándose un instrumento, que contemplaba criterios evaluativos validados por 11 especialistas. Resultados: En las consultas observadas, las enfermeras implementaron esas acciones de forma adecuada, careciendo, no obstante, de complementaciones. Conclusión: El proceso asistencial, a pesar de ser desarrollado adecuadamente, requiere de ajustes de fácil resolución en algunos procedimientos, a fin de abarcar aspectos esenciales para la salud del niño en las unidades seleccionadas del Programa de Salud de la Familia.

Descriptores: Evaluacion de Servicios de Salud; Servicios Básicos de Salud; Calidad de la Atención de Salud; Salud del Niño; Salud de la familia; Remisión y consulta

* Artigo extraído da Tese de Doutorado "Avaliação da qualidade da consulta de enfermagem à criança em Unidades Básicas de Saúde no Programa de Saúde da Familia", apresentada ao Programa de Pós-Graduação em Enfermagem da Universidade Federal de São Paulo - UNIFESP - São Paulo (SP), Brasil.

${ }^{1}$ Professor Doutor do Departamento de Enfermagem da Universidade Federal de São Paulo - UNIFESP - São Paulo (SP), Brasil.

${ }^{2}$ Professora Titular do Departamento de Enfermagem da Universidade Federal de São Paulo - UNIFESP - São Paulo (SP), Brasil.
\end{abstract}




\section{INTRODUÇÃO}

A consulta de enfermagem, assegurada pela legislação de Enfermagem, deve ser repensada na perspectiva do Programa de Saúde da Família (PSF) que visa aprimorar e assegurar os princípios do Sistema Único de Saúde. A aplicação desta estratégia possibilita reorganizar a assistência básica à saúde para desenvolver ações de promoção, proteção e recuperação da saúde do indivíduo, da família e da comunidade; identificar precocemente a necessidade de tratamento das doenças, por meio da atuação de equipes de saúde, de caráter multiprofissional, capacitadas tecnicamente a prestar assistência integral resolutiva, contínua e de boa qualidade, para atender as necessidades de saúde da população cadastrada e atendida na unidade de saúde e na comunidade ${ }^{(1)}$.

A implementação do PSF promoveu o avanço da implantação da consulta de enfermagem em Unidades Básicas de Saúde, devido à contratação de maior número de enfermeiras que realizam esta atividade, de forma contínua, aos usuários destas unidades, constituindo uma estratégia de atendimento de caráter generalista, centrada no ciclo vital e na assistência à família.

Esta prática assistencial, inserida no processo do trabalho coletivo em saúde, possibilita diagnosticar necessidades de saúde, prescrever e prestar cuidados de enfermagem resolutivos e qualificados ${ }^{(2)}$.

Esta ação integrada na programação local é habitualmente intercalada com a consulta médica, segundo cronogramas de atendimentos estabelecidos nos serviços de saúde, contribuindo para a melhoria da qualidade da assistência prestada à criança, visando à promoção, proteção e recuperação da saúde desta clientela $^{(3-4)}$.

Para que seja prestada com qualidade, é fundamental que os serviços de saúde disponham de estruturas adequadas no que se refere à área física e instalações, disponibilidade de materiais e equipamentos, número adequado de enfermeiras com capacitação específica, que interajam com o cliente e família na perspectiva da criação de vínculo e respeito à autonomia do usuário.

Diante destas considerações, aliadas à vivência de uma das pesquisadoras, procurou-se, na pesquisa de doutorado ${ }^{(5)}$ obter respostas a algumas perguntas relacionadas à qualidade da consulta de enfermagem selecionando-se, para este trabalho, a seguinte questão: as ações básicas de saúde propostas pelo Ministério da Saúde são implementadas de forma adequada nas consultas de enfermagem prestadas às crianças em Unidades Básicas de Saúde?

A investigação supracitada foi ancorada na concepção sistêmica da avaliação de serviços de saúde e, para este recorte, selecionou-se a abordagem de processo, focando a dimensão da aplicação de conhecimentos técnicocientíficos $^{(\sigma)}$ na prestação da consulta de enfermagem.

Desta forma, este estudo objetivou avaliar a qualidade da consulta de enfermagem prestada à criança até um ano de idade em Unidades Básicas de Saúde no PSF do Município de São Paulo, relacionada à aplicação de conhecimentos técnico-científicos na implementação das ações básicas de saúde propostas pelo Ministério da Saúde.

\section{MÉTODOS}

Trata-se de um estudo do tipo descritivo e avaliativo ${ }^{(7-8)}$ e, para seleção do campo de estudo e população a ser investigada, foram estabelecidos os seguintes critérios de inclusão: Unidades Básicas de Saúde (UBS) do Projeto QUALIS implantadas no período de 1996 a 1998, época do início do PSF na capital paulistana; e, enfermeiras contratadas neste triênio, capacitadas por profissionais da mesma categoria, para prestar a consulta de enfermagem à criança nesses serviços de saúde.

Assim, a população do estudo abrangeu 14 enfermeiras que realizavam a consulta de enfermagem à criança até um ano de idade em oito UBS do PSF em parceria com a Casa de Saúde Santa Marcelina, localizadas na zona Leste da capital paulistana. A amostra incluiu $20,0 \%$ do total de crianças dessa faixa etária cadastradas na equipe de saúde de cada enfermeira, abrangendo 114 consultas de enfermagem.

Os dados foram coletados, no período de março a junho de 2002 por meio da observação sistematizada do processo desenvolvido na consulta de enfermagem, orientada por um instrumento, contemplando a aplicação de conhecimentos técnico-científicos na implementação das cinco ações básicas de saúde: acompanhamento do crescimento e desenvolvimento infantil, promoção do aleitamento materno e orientação para o desmame, controle das doenças imunopreveníveis por vacinas, assistência e controle das infecções respiratórias agudas e controle das doenças diarréicas.

Para proceder à avaliação proposta, foi construído o referido instrumento abrangendo critérios normativos extraídos da literatura consultada ${ }^{(9-20)}$, que foi validado por 11 especialistas nas áreas de Saúde Pública/Saúde Coletiva e Saúde da Criança.

Precedendo a coleta de dados, o projeto de pesquisa foi aprovado pelo Comitê de Ética em Pesquisa da Universidade Federal de São Paulo (Processo no 564/01) e, pela Coordenação de Enfermagem do então QUALIS Santa Marcelina. As enfermeiras selecionadas concordaram em participar do estudo após serem devidamente informadas acerca dos objetivos do estudo e sobre a técnica de observação sistematizada, assinando o Termo de Consentimento Livre e Esclarecido.

\section{RESULTADOS}

\section{Acompanhamento do crescimento e}




\section{desenvolvimento da criança}

Em relação ao acompanhamento do crescimento da criança, observou-se que em todas as consultas, as enfermeiras participantes do estudo mensuraram o peso e comprimento da criança.

Quanto aos registros das principais medidas antropométricas no Gráfico de Acompanhamento do Crescimento contido no prontuário, na grande maioria das consultas $(86,0 \%)$ essas profissionais anotaram o peso e comprimento. Verificou-se que, dentre os atendimentos nos quais foi mensurado o perímetro cefálico $(79,8 \%)$, em apenas $9,8 \%$ esta medida foi registrada no gráfico.

Além disto, em cerca de 96,7\% destas consultas as crianças foram pesadas segundo os procedimentos indicados com a participação dos acompanhantes, o mesmo acontecendo por ocasião da mensuração do comprimento em 90,4\% dos atendimentos.

Após análise das curvas de crescimento, as enfermeiras identificaram em apenas $5,2 \%$ das consultas, que as crianças encontravam-se com peso situado acima ou abaixo da faixa de normalidade preconizada pelos padrões de referência utilizados, indicando uma situação nutricional favorável para a grande maioria das crianças atendidas. Verificou-se, ainda, que as mães ou responsáveis pela criança foram orientadas adequadamente com vistas à recuperação do peso, segundo as situações identificadas.

Quanto ao registro do peso no Cartão da Criança, em $84,2 \%$ das consultas, foi anotada esta medida na curva de crescimento, e em 12,3\% e 3,5\% das atividades, respectivamente, o peso não foi registrado devido, tanto ao esquecimento das mães/responsáveis de trazer o Cartão, como pela falta de registro desta mensuração.

Partindo da premissa que os marcos universais do desenvolvimento infantil esperados no primeiro ano de idade, recomendados por alguns testes e escalas, não diferem muito entre si, utilizou-se, neste estudo, como padrão de referência para a avaliação, a adaptação dos principais marcos estabelecidos por esses instrumentos, destacando-se, dentre eles, a Ficha de Acompanhamento do Desenvolvimento, adotada nas UBS e por outras referências bibliográficas consultadas, a respeito dos parâmetros do desenvolvimento infantil.

Dentre as 114 consultas observadas, em 103 (79,0\%) as enfermeiras identificaram de forma completa $(30,7 \%)$ ou quase completa $(48,3 \%)$ os itens referentes aos principais marcos do desenvolvimento esperado no primeiro ano de vida da criança, sendo que esta identificação foi completa em 82,3\% dos atendimentos realizados às crianças no primeiro mês de vida. Porém, no que tange à orientação, em 93 consultas $(81,2 \%)$ ocorreu diálogo com as mães/responsáveis sobre o processo do desenvolvimento infantil, orientando-as sobre medidas que favorecem a aquisição das habilidades esperadas para suas crianças.
Também, nas 114 consultas observadas (100\%) foi enfatizada a importância do acompanhamento do crescimento e desenvolvimento infantil realizado pela equipe de saúde, solicitando à família o comparecimento da criança conforme agendamento de rotina que estabelece a intercalação entre as consultas de enfermagem e médica.

\section{Promoção do aleitamento materno e orientação alimentar por ocasião do desmame}

Nas consultas observadas, em mais da metade dos atendimentos $(66,0 \%)$ em que as crianças tinham até quatro meses de idade, o leite materno era oferecido exclusivamente.

Quanto ao grau de satisfação das mães e dificuldades encontradas para amamentar seus filhos, dentre os 17 atendimentos prestados às crianças com menos de dois meses, em cerca de dois terços destas consultas foram avaliadas estas condições, e as mães foram orientadas com vistas a superar dificuldades.

Observou-se ainda que destas 17 consultas prestadas, em menos da metade delas $(41,2 \%)$ foram avaliadas as condições das mamas das mulheres que amamentavam, e apenas uma delas apresentou ingurgitamento e fissuras mamárias sem, contudo, ser orientada para o tratamento específico.

No entanto, em relação às orientações sobre as condutas indicadas para amamentação eficaz da criança, observou-se que em 64,7\% dos atendimentos prestados às crianças de até dois meses de idade, estas condutas foram discutidas com as mães/responsáveis.

Um outro fator que demonstra a adequada ingestão de leite materno é o ganho satisfatório de peso da criança durante o primeiro ano de vida; contudo, somente em um atendimento este cálculo foi realizado.

Dentre os 15 atendimentos em que as crianças, menores de quatro meses, estavam em aleitamento misto ou artificial, em apenas três $(20,0 \%)$, houve orientações para a relactação..

Estes resultados foram considerados pouco satisfatórios em função de grande parte $(80,0 \%)$ das crianças menores de quatro meses, que estavam desmamadas, não terem sido relactadas $(n=12)$, sobretudo em quatro delas, com até dois meses, cujo processo de relactação poderia ter maior sucesso.

Nas 79 consultas, em que as crianças encontravam-se em aleitamento misto ou artificial, as enfermeiras identificaram o tipo, concentração e preparo do leite. Porém, em quase metade deles, o leite em pó integral $(n=32)$ ou modificado para uso infantil $(n=5)$ era oferecido; nestes casos, em apenas três consultas $(8,1 \%)$, as mães/ responsáveis foram orientadas sobre a concentração adequada, conforme idade em meses das crianças atendidas.

Controle das doenças imunopreveníveis por vacinas 
Neste estudo, verificou-se que as 114 crianças (100\%) atendidas nas consultas de enfermagem tinham tomado as vacinas indicadas para a idade, mediante informação obtida, quer no Cartão da Criança $(87,7 \%)$, quer pelo relato das mães/acompanhantes (12,3\%) que alegaram ter esquecido este documento. Para estas, as enfermeiras reforçaram a importância da família apresentá-lo em todos os atendimentos realizados nos serviços de saúde.

Quanto às orientações sobre as vacinas, essas profissionais enfatizaram a necessidade da administração desses imunobiológicos para proteção contra as doenças evitáveis pela imunização, em 98,2\% dos atendimentos. No entanto, em $64,9 \%$ das consultas observou-se que as mães/acompanhantes foram informadas sobre os tipos e doses de vacinas recomendadas, e apenas em 7,0\% dos atendimentos foram esclarecidas sobre as possíveis reações locais e sistêmicas que podem ocorrer após a vacinação. Mas em nenhuma consulta as enfermeiras orientaram às mães/responsáveis para retornarem ao serviço, caso a criança apresentasse eventos adversos.

\section{Controle das Doenças Diarréicas da Infância}

Em todas as consultas, as mães/acompanhantes foram questionadas acerca da presença ou não de diarréia, identificando-se apenas uma criança com três meses e quinze dias de idade, em aleitamento artificial, que apresentava diarréia sem desidratação e também obstrução e secreção nasal.

Após avaliação do seu estado geral, a enfermeira orientou a mãe a manter a alimentação, aumentar a oferta de líquidos e retornar ao serviço se a criança piorasse. No entanto, o oferecimento de solução reidratante oral, com vistas a manter o estado de hidratação desta criança, não foi informado à mãe, como é recomendado.

\section{Assistência e Controle das Infecções Respiratórias Agudas}

Nas consultas de enfermagem observadas, as enfermeiras verificaram se havia presença de tosse ou dificuldade respiratória, assim como possíveis sinais de gravidade que demandavam busca imediata de atendimento hospitalar. Nas crianças atendidas, os sinais e sintomas de maior freqüência foram a secreção nasal $(43,0 \%)$ e a tosse $(28,9 \%)$. Nas 33 crianças $(28,9 \%)$ que apresentavam tosse, foi identificado o tempo de duração deste sinal na maioria das consultas $(72,7 \%)$, sendo que em todas elas a presença ou não de estridor e sibilância foi observada, conforme protocolo proposto para o atendimento às infecções respiratórias agudas. Quanto à verificação da freqüência respiratória, dessas 33 crianças, em apenas 12 (36,4\%) esta medida foi realizada, embora tenha sido verificada em um minuto e com a criança tranqüila, em apenas sete atendimentos $(21,2 \%)$.

$\mathrm{Na}$ consulta prestada a uma criança de um mês e quinze dias de vida, em aleitamento artificial, foi verificada a gravidade do seu estado por apresentar tosse, aumento da freqüência respiratória, dispnéia acompanhada de tiragem subcostal e presença de roncos e crepitações. A conduta da enfermeira foi a de solicitar confirmação diagnóstica pelo médico da equipe que a encaminhou a um pronto-socorro próximo da unidade, e solicitou retorno para avaliação da evolução da infecção respiratória. Por se tratar do primeiro atendimento realizado nesse serviço, esta criança, por não ser acompanhada rotineiramente em UBS da região, apresentou agravo do seu quadro respiratório devido ao fato de não ter sido atendida precocemente por profissionais de saúde.

Ressalta-se que dentre as 58 consultas em que as crianças apresentaram sinais e/ou sintomas respiratórios isolados ou associados, as profissionais avaliaram em apenas 15 deles $(25,8 \%)$ as condições de moradia da família.

As orientações gerais de enfermagem que auxiliam a manutenção do estado de hidratação da criança e a fluidificação de secreções, bem como os cuidados com o ambiente doméstico foram condutas adotadas em poucos atendimentos em que as crianças apresentaram sinais e sintomas respiratórios $(33,3 \%, 19,7 \%, 12,1 \%$, respectivamente). Ocorreu o mesmo na indicação para as mães evitarem o contato da criança com poeiras, fumaça de cigarro e outros alergenos $(21,2 \%)$.

\section{DISCUSSÃO}

O Programa de Assistência Integral à Saúde da Criança (PAISC) prioriza as cinco ações básicas de saúde contempladas neste estudo, que apresentam comprovada eficácia e reduzida complexidade tecnológica, para reduzir os agravos mais freqüentes e de maior relevância na morbi - mortalidade de crianças até cinco anos de vida.

\section{Acompanhamento do crescimento e desenvolvimento da criança}

O crescimento constitui um dos melhores indicadores de saúde da criança em função de sua estreita dependência de fatores sociais relacionados ao: tipo de alimentação em quantidade e qualidade; ocorrências e tipos de doenças; cuidados de saúde e vacinação; afetividade da família com a criança; condições de vida, destacando-se a moradia, o saneamento básico e o acesso aos serviços de saúde $e^{(9)}$.

Os resultados desta pesquisa, referentes à mensuração do peso e comprimento da criança $(100,0 \%)$, ao registro destas medidas no Gráfico de Acompanhamento do Crescimento $(86,0 \%)$ e à anotação do peso no Cartão da Criança $(84,2 \%)$ foram animadores, especificamente quanto a este último item, quando comparados com os 
dados do inquérito da Pesquisa Nacional de Demografia e Saúde - PNDS/1996. Este estudo ${ }^{(9)}$ demonstrou que a maioria das mães não levava o referido Cartão às consultas, sendo que menos de $10 \%$ destes apresentavam o registro do peso e menor percentagem ainda, tinha a curva de crescimento desenhada no gráfico do Cartão, o que evidenciou sua baixa utilização para monitorar o crescimento infantil pelos profissionais de saúde.

Uma possível explicação para a alta percentagem do registro de peso na curva do Cartão da Criança, obtida nesta pesquisa, é a implementação do PSF nas UBS estudadas, que enfatiza a adoção desta medida pela equipe de saúde da família.

O desenvolvimento caracteriza-se como um processo global e dinâmico de mudanças que ocorrem em uma pessoa, desde sua concepção, influenciado por diversos fatores físicos, dentre eles, os cuidados com a alimentação, a higiene e a prevenção de doenças, além da dimensão psicológica, relacionada à forma como uma criança aprende e interage com o ambiente em que vive ${ }^{(10)}$.

A enfermeira deve conhecer o processo de desenvolvimento infantil e utilizar algum instrumento como recurso para esta avaliação. É recomendado que ao se observar as diversas áreas do desenvolvimento infantil, se considere a existência de uma seqüência ordenada para a aquisição de habilidades, mas não uma velocidade padrão, justificando, assim, o fato das crianças poderem apresentar diferenças nos marcos do desenvolvimento esperados, segundo a idade exata ${ }^{(17)}$.

Sendo assim, a avaliação adequada do desenvolvimento infantil deve ser ampla, requerendo durante a entrevista, a coleta de informações sobre as condições de vida, rotina diária, formas de relacionamento familiar e oportunidades de interação com outras crianças, pois para desenvolver uma determinada habilidade, ela deve vivenciar situações que favoreçam a aquisição da mesma $^{(17)}$.

Porém, as profissionais participantes do estudo não utilizaram testes e escalas para a avaliação das crianças, identificando alguns dos principais marcos do desenvolvimento esperado no primeiro ano de vida, a partir do seu conhecimento e experiência sobre o processo do desenvolvimento infantil. No transcorrer das consultas, observaram aquilo que a criança já realizava, assim como sua interação com a mãe/ responsável e outras crianças que, na ocasião, as acompanhavam, oferecendo algumas vezes brinquedos e outros objetos adequados que auxiliam na avaliação da aquisição de determinadas habilidades esperadas para cada uma delas.

Assim, acredita-se ser indicada, a utilização de um roteiro de observação na consulta de enfermagem que contemple os principais dados para guiar essa avaliação, para evitar a omissão da identificação dos principais marcos esperados no desenvolvimento infantil no primeiro ano de vida.

Portanto, o acompanhamento sistemático do crescimento e desenvolvimento da criança menor de cinco anos, em especial até o segundo ano de vida, é recomendado em função da vulnerabilidade biológica nesta faixa etária, podendo diferentes crianças com a mesma idade apresentar graus de vulnerabilidade e riscos de adoecer e morrer completamente distintos, segundo as condições e qualidade de vida de suas famílias ${ }^{(9)}$.

\section{Promoção do aleitamento materno e orientação alimentar por ocasião do desmame}

O aleitamento materno é importante tanto para a prevenção de doenças infecciosas, especialmente, das enfermidades diarréicas, como para o crescimento e desenvolvimento da criança que é bastante dependente da satisfação das necessidades nutricionais. É consenso que as condições adequadas de alimentação implicam na oferta exclusiva de leite materno durante, pelo menos, quatro meses e, se possível, nos seis meses de vida, além da complementação por outros alimentos a partir desta idade $^{(9,19)}$

No Brasil, inquéritos nacionais têm demonstrado que a amamentação por tempo prolongado é pouco freqüente, sendo menor ainda a freqüência da oferta exclusiva de leite materno nos primeiros meses de vida ${ }^{(20)}$.

Contudo, verificou-se neste estudo que em mais da metade dos atendimentos $(66,0 \%)$ às crianças até quatro meses de idade, o leite materno era oferecido exclusivamente, correspondendo a um percentual um pouco superior ao alcançado pelas UBS do PSF em parceria com a Casa de Saúde Santa Marcelina (62,4\%), e acima da média da capital paulistana (40,0\%), segundo dados de $2001^{(20)}$.

Quanto à técnica adequada para a amamentação, esta orientação foi omitida em alguns atendimentos $(35,3 \%)$ prestados à criança com até dois meses de idade, supondose que esta omissão foi pelo fato das enfermeiras indicarem estas condutas nas visitas domiciliarias aos recém-nascidos, o que não impediria o reforço dessa orientação nessas consultas, a fim de prevenir o desmame precoce.

Considerando-se os resultados referentes à adoção desta ação básica de saúde, percebeu-se uma tendência das enfermeiras participantes deste estudo a oferecer orientações para que as mães amamentassem seus filhos de forma eficiente em $64,7 \%$ das consultas prestadas às crianças com até dois meses de idade, assim como indicar a introdução adequada de alimentos não-lácteos, em quantidade e qualidade, condizentes com as condições sócio-econômicas das suas famílias, nas 64 consultas prestadas às crianças a partir de quatro meses de idade em aleitamento misto e artificial.

Em contrapartida, a orientação sobre a concentração adequada de leite artificial, verificada somente em três 
atendimentos e de hidratos de carbono $(26,6 \%)$ oferecida às mães/responsáveis pelas crianças que estavam em situação de desmame, não fez parte da conduta estabelecida por essas profissionais, o que pode acarretar uma oferta inferior ou superior às necessidades da criança, além da sobrecarga de solutos.

Portanto, a qualidade da consulta de enfermagem no que tange a esta ação básica de saúde deveria ser melhorada por requerer alguns ajustes de simples resolução referentes ao: reforço das orientações, quando indicado, acerca da técnica correta para amamentação; preparo e cuidados com as mamas e mamilos para a oferta do leite materno; procedimento para a avaliação da efetividade da amamentação (avaliação do ganho de peso diário da criança); orientação para ordenha mamária e relactação; verificação e orientação adequadas da concentração do leite artificial e oferta de hidratos de carbono às crianças desmamadas.

\section{Controle das doenças imunopreveníveis por vacinas}

As vacinas recomendadas pelo MS e Secretarias Estaduais de Saúde são indicadas segundo a situação epidemiológica do local e disponibilidade de recursos da região, a fim de prevenir determinadas doenças infecto-contagiosas, sendo recomendado manter altas coberturas vacinais e evitar oportunidades perdidas nos serviços de saúde ${ }^{(11)}$.

Neste estudo, todas as crianças atendidas estavam com o esquema vacinal completo para a idade, sendo que estes resultados eram próximos aos alcançados pelas UBS do PSF em parceria com a Casa de Saúde Santa Marcelina, atingindo $95,0 \%$ de crianças menores de um ano com o esquema vacinal completo, conforme dados de $2001^{(20)}$.

Embora as mães/responsáveis por essas crianças recebam orientação na sala de imunização, acredita-se que seria desejável que as enfermeiras, na consulta de enfermagem, verificassem o conhecimento das mesmas a respeito da vacinação para melhor adequação das atividades educativas.

\section{Controle das Doenças Diarréicas da Infância}

Este controle tem sido uma das ações que contribuem para a redução da mortalidade causada por diarréias em crianças menores de cinco anos, destacando-se duas estratégias importantes: o aleitamento materno e o saneamento ambiental, sobretudo a qualidade da água ${ }^{(12)}$.

Um dos motivos que pode justificar a presença de diarréia em apenas uma criança consultada foi à incidência de $66,0 \%$ de aleitamento materno exclusivo em crianças até quatro meses de idade, acrescido ao fato de 11,3\% delas estarem em aleitamento misto, perfazendo um total de $71,3 \%$ de crianças nesta faixa etária que recebiam leite materno, o que reforça a afirmação de que a prática do aleitamento materno é um fator de proteção na infância contra as doenças diarréicas e suas complicações.

Outro motivo de destaque é o fato das crianças atendidas serem continuamente acompanhadas pela equipe de saúde, tanto nas unidades como nos domicílios, supondo-se que suas famílias recebiam orientações sobre prevenção e cuidados com a criança durante episódios diarréicos, embora a administração do soro reidratante oral no domicílio não tenha sido indicada em um atendimento, segundo normas do Ministério da Saúde e da Estratégia Atenção Integrada às Doenças Prevalentes da Infância (AIDPI) ${ }^{(13-14)}$.

Neste estudo, percebeu-se que no atendimento à criança com diarréia a enfermeira demonstrou conhecimento sobre as condições de vida de sua família; contudo não relacionou estas condições com a adoção de medidas para a prevenção deste agravo como: a qualidade da água utilizada no preparo do leite e a adequação dos cuidados higiênicos necessários ao preparo e acondicionamento da mamadeira.

\section{Assistência e Controle das Infecções Respiratórias Agudas}

As infecções respiratórias agudas (IRA) constituem um dos principais problemas de saúde nas crianças menores de cinco anos em países em desenvolvimento. No Brasil, desde 1984, o MS vem desenvolvendo ações de controle destas infecções para reduzir a morbi-mortalidade, sobretudo por meio do diagnóstico precoce e do tratamento eficaz dos casos diagnosticados. Apesar do decréscimo deste grupo de doenças na mortalidade infantil, representam, ainda, a primeira causa de internação em crianças menores de um ano de idade nos serviços públicos de saúde, sendo a pneumonia a principal causa de óbito ${ }^{(14)}$.

Dentre as 33 crianças que apresentavam tosse, as enfermeiras verificaram a presença ou não de estridor e sibilância em todos os atendimentos e na maioria das consultas $(72,7 \%)$ o tempo de duração da tosse foi identificado conforme proposto pela estratégia AIDPI $^{(13)}$.

Quanto à verificação da freqüência respiratória durante um minuto ${ }^{(13)}$, com a criança tranqüila, quando a mesma apresentar tosse ou dificuldade respiratória, em apenas sete atendimentos $(21,2 \%)$ essa medida foi aferida nestas condições, o que foi considerado pouco satisfatório.

Face aos resultados encontrados, percebeu-se que as enfermeiras enfatizaram as orientações para resolução de alguns sinais e sintomas respiratórios, orientando em poucos atendimentos as principais medidas de prevenção destes problemas sem, contudo, relacioná-los com as condições de moradia da clientela atendida.

\section{CONCLUSÃO}

A qualidade do processo assistencial desenvolvido 
pelas 14 enfermeiras, participantes desta pesquisa, nas 114 consultas de enfermagem prestadas às crianças até um ano de idade foi considerada relativamente adequada, no que tange à aplicação de conhecimentos técnico-científicos na implementação das cinco ações básicas de saúde preconizadas pelo Ministério da Saúde. No entanto, alguns procedimentos, relacionados principalmente aos aspectos educativos, requerem ajustes de fácil resolução, a fim de contemplar aspectos essenciais à saúde da criança, aprimorando, assim, a qualidade da consulta de enfermagem prestada em Unidades Básicas de Saúde no Programa de Saúde da Família da cidade de São Paulo.

\section{REFERÊNCIAS}

1. Brasil. Ministério da Saúde. Secretaria de Assistência à Saúde. Saúde da família: uma estratégia para a reorientação do modelo assistencial. 2a ed. Brasília: Ministério da Saúde; 1998.

2. Nielsen GH, Mortensen R. Classificação Internacional das Práticas de Enfermagem do Conselho Internacional de Enfermeiras: versão Alpha. Traduzido por Cruz DALM, Carvalho EC, Marin HF, Nóbrega ML. Brasília: Associação Brasileira de Enfermagem; 1997. [Série Didática; Enfermagem no SUS].

3. São Paulo.Secretaria de Estado da Saúde. Centro de Apoio ao Desenvolvimento de Assistência Integral à Saúde. Grupo de Coordenação para Assuntos de Enfermagem. Consulta de enfermagem: conceitos e componentes básicos. São Paulo;1992.

4. Ribeiro CA, Silva CV, Saparolli ECL. Consulta de enfermagem à criança num projeto de integração docenteassistencial: experiência de implantação. Acta Paul Enfermagem. 2002;15(2):79-88.

5. Saparolli ECL. Avaliação da qualidade da consulta de enfermagem à criança em unidades básicas de saúde do programa de saúde da família [tese]. São Paulo: Universidade Federal de São Paulo; 2003.

6. Donabedian A. The definition of quality and approaches to its assessment. An Arbor, Mich.: Health Administration Press; c1980. [Série: His Explorations in quality assessment and monitoring, 1]

7. Aguilar MJ, Ander-Egg E. Avaliação de serviços e programas sociais. Petrópolis: Vozes; 1994.

8. Cervo AL, Bervian PA. Metodologia científica. 4a ed. São Paulo: Makron Books Ed.; 1996.
9. Brasil. Ministério da Saúde. Secretaria de Políticas de Saúde. Área Técnica de Saúde da Criança. Área Técnica de Alimentação e Nutrição. Fundamentos técnico-científicos e orientações práticas para o acompanhamento do crescimento e desenvolvimento. Parte 1- Crescimento. Brasília: Ministério da Saúde; 2000.

10. Brasil.Ministério da Saúde. Fundação Nacional da Saúde. O que podemos fazer juntos: desenvolvimento global e atividades da criança até 3 anos. 3 a ed. Brasília: Ministério da Saúde; 1994.

11. Bricks LF, Oselka GW. Imunizações. In: Marcondes E, Vaz FAC, Ramos JLA, Okay Y, coordenadores. Pediatria Básica: Pediatria geral e neonatal. 9a ed. São Paulo: Sarvier; 2002. t. 1. p.102-13.

12. Brasil.Ministério da Saúde. Secretaria de Assistência à Saúde. Coordenação de Saúde Materno-Infantil. Assistência e Controle das Doenças Diarréicas. Brasília (DF)Ministério da Saúde; 1993.

13. Brasil.Ministério da Saúde.Organização Mundial da Saúde.Organização Pan-Americana da Saúde. Atenção Integrada às Doenças Prevalentes da Infância: quadro de conduta. Brasília (DF):Ministério da Saúde; 1999.

14. Marcondes E, Vaz FAC, Ramos JLA, Okay Y, coordenadores. Pediatria Básica: Pediatria geral e neonatal. 9a ed. São Paulo: Sarvier; 2002. t. 1.

15. São Paulo. Secretaria de Estado da Saúde. Centro de Apoio ao Desenvolvimento de Assistência Integral à Saúde (CADAIS). Manual de orientações para aleitamento materno, alimentação da criança no primeiro ano de vida, da gestante e do adolescente. São Paulo; 1992.

16. Whaley LF, Wong DL. Enfermagem pediátrica: elementos essenciais à intervenção efetiva. 5a ed. Traduzido por Araújo CLC, Mundim FD, Cabral IE, Figueiredo JEF, Couto LB, Voeux PJ. Rio de Janeiro: Guanabara Koogan; 1999.

17. Sucupira ACSL, Werner JJ, Resegue R. Desenvolvimento. In: Sucupira ACSL, Bricks LF, Kobinger MEBA, Saito MI, Zuccolotto SMC, coordenadores. Pediatria em consultório. 4a ed. São Paulo: Sarvier; 2000. p.22-39.

18. Marcondes E. Crescimento. In: Sucupira ACSL, Bricks LF, Kobinger MEBA, Saito MI, Zuccolotto SMC, coordenadores. Pediatria em consultório. 4a ed. São Paulo: Sarvier; 2000. p.16-21.

19. Bresolin AMB, Lima IN, Penna HAOP, Issler H. Alimentação da criança. In: Marcondes E, Vaz FAC, Ramos JLA, Okay Y, coordenadores. Pediatria Básica: Pediatria geral e neonatal. 9a ed. São Paulo: Sarvier; 2002.t. 1, p.61-95

20. São Paulo. Secretaria de Estado da Saúde. $6^{\circ}$ aniversário QUALIS: muita saúde para você. São Paulo; 2002. 